\title{
Development of a coupler h3-Open-UTIL/MP
}

\author{
Takashi Arakawa* \\ arakawa@rist.jp \\ Reseach Organization for Information \\ Science and Technology \\ Minato-ku, Tokyo, Japan
}

\author{
Hisashi Yashiro* \\ yashiro.hisashi@nies.go.jp \\ National Institute for Environmental \\ Studies \\ Tsukuba, Ibaraki, Japan
}

\author{
Kengo Nakajima \\ Information Technology Center, the \\ University of Tokyo \\ Bunkyo-ku, Tokyo, Japan \\ nakajima@cc.u-tokyo.ac.jp
}

\begin{abstract}
In this work, we develop a coupler, h3-Open-UTIL/MP, as part of the h3-Open-BDEC project. The h3-Open-UTIL/MP coupler has an ensemble coupling capability to run multiple coupled programs in parallel and an interface to enable Python application coupling. In this paper, first, we outlined the features of h3-Open-UTIL/MP and its ensemble coupling capabilities. Then, we reported the details of Python application coupling and a coupling case study of the atmospheric model NICAM using a machine learning (ML) framework. To couple a Python application, we added a Python wrapper to the h3-Open-UTIL/MP interface provided as a Fortran module. Subsequently, we load Fortran routines as a library in the Python application. We used the ML framework PyTorch as a coupling example. We targeted the NICAM cloud physics subroutine and trained it to generate output variables from input variables. The results show a room for improvement in terms of reproducibility. Reproducibility can be improved in several ways: data selection, preprocess improvement, and changes in ML algorithms. In addition, the ML perspective was the bottleneck of execution time. Hence, there is a clear need to improve the computational performance. One effective method to improve computational performance is to run NICAM on a conventional high-performance computer and PyTorch on a GPU machine. We are currently developing a heterogeneous machine coupling program to implement this coupling method, which is briefly described in the final section of this paper.
\end{abstract}

\section{CCS CONCEPTS}

\section{- Computing methodologies $\rightarrow$ Simulation tools}

\section{KEYWORDS}

coupler, atmospheric model, neural networks, heterogeneous system

\section{ACM Reference Format:}

Takashi Arakawa, Hisashi Yashiro, and Kengo Nakajima. 2022. Development of a coupler h3-Open-UTIL/MP. In International Conference on High Performance Computing in Asia-Pacific Region (HPC Asia2022), January 12-14, 2022, Virtual Event, fapan. ACM, New York, NY, USA, 12 pages. https://doi.org/10.1145/3492805.3492809

Permission to make digital or hard copies of all or part of this work for personal or classroom use is granted without fee provided that copies are not made or distributed for profit or commercial advantage and that copies bear this notice and the full citation on the first page. Copyrights for components of this work owned by others than ACM must be honored. Abstracting with credit is permitted. To copy otherwise, or republish, to post on servers or to redistribute to lists, requires prior specific permission and/or a fee. Request permissions from permissions@acm.org.

HPC Asia2022, January 12-14, 2022, Virtual Event, Japan

(C) 2022 Association for Computing Machinery.

ACM ISBN 978-1-4503-8498-8/22/01 ..\$15.00

https://doi.org/10.1145/3492805.3492809

\section{INTRODUCTION}

A purpose of numerical simulations is to faithfully reproduce phenomena to facilitate the understanding of the subject matter and to make more precise predictions. Despite the varying objectives of the simulation depending on the field and purpose, coupling multiple phenomena with different spatio-temporal resolutions may be required. In the field of atmospheric research, weather/climate simulations are routinely carried out. In this field, a computational method has been used wherein an atmospheric model and an ocean model perform calculations at their own spatio-temporal resolutions, exchanging data with each other at appropriate time intervals (e.g., the MRI-GCM of the Meteorological Research Institute in Japan[16]). Recently, various research institutes have been developing Earth System Models (ESMs) that incorporate many physical and chemical factors to achieve more precise climate simulations. One example is the MIROC-ESM being developed by the Japan Agency for Marine Earth Science and Technology, in collaboration with the National Institute of Environmental Studies, Atmosphere Ocean Research Institute of the University of Tokyo, and a community of other universities (Team MIROC)[8]. When multiple model components are coupled, "couplers" are generally used as a software layer to determine the differences in spatio-temporal resolution and to exchange data at appropriate time intervals. With this background, various coupling software and software frameworks for coupling have been developed. For example, ESMF is a software framework for earth system modeling[6]. ESMF provides several functions for various component models that make up the earth system model, and it can flexibly couple component models that have various grid systems. Furthermore, a software suite ESPS (i.e., the Earth System Prediction Suite) has been developed with ESMF at its core for unified handling of various model components for climate simulation[13]. ESMF and ESPS are fat-approach software frameworks because they provide various functions, such as execution control with a focus on coupling functions. Conversely, OASIS3-MCT[3] and YAC[5], which are widely used in European research institutes, are considered thin-approach software because they specialize in coupling functions only. In Japan, Scup was developed as a thin-approach coupler by Yoshimura and Yukimoto and is used in the Earth System Model of the Meteorological Research Institute[15].

The primary focus of these coupling software is weather/climate simulations. Meanwhile, in this study, we develop the h3-OpenUTIL/MP coupler to be used in a broader range of fields and on stateof-the-art computers. The h3-Open-UTIL/MP coupler was started in 2019 and developed as part of the h3-Open-BDEC project[9]. The h3-Open-BDEC (i.e., h3: hierarchical, hybrid, heterogeneous) is an open source infrastructure for the development and execution of optimized and reliable codes for big data \& extreme computing 
(BDEC). Fig. 1 shows the structure of the h3-Open-BDEC software suite. The software suite consists of eight software packages, such as h3-Open-MATH and h3-Open-DATA; in particular, h3-OpenUTIL/MP is part of the large-scale computing utility package h3Open-UTIL.

In this paper, we report on the features and application examples of h3-Open-UTIL/MP. The remainder of this paper is organized as follows. Section 2 briefly describes the API and features of h3Open/UTIL/MP, and Section 3 discusses the function of ensemble coupling, which is one of the features of the coupler. In Section 4, we report on the coupling of Python applications. In this section, we first describe the structure of the program and the API for Python and then describe in detail the coupling of the atmospheric model NICAM with the machine learning (ML) library as a case study. In the concluding section, we mention our ongoing $R \& D$ program on heterogeneous machine coupling in collaboration with other teams of h3-Open-BDEC.

\section{OVERVIEW OF H3-OPEN-UTIL/MP}

One of the important features of h3-Open-UTIL/MP coupler is that it has been developed as one part of the h3-Open-BDEC project \& software suite. By linking with other parts of the h3-Open-BDEC software suite, h3-Open-UTIL/MP will come to be able to perform advanced coupled calculations that cannot be achieved by conventional coupling software, such as coupled calculations on heterogeneous computer systems. However, the linking program with other parts remains under development, and its will be discussed in the conclusion. In this section, we describe the basic features of h3-Open-UTIL/MP alone, which is to exchange data between model components with different grid systems at the appropriate timing while absorbing the differences between the grids. The advanced features of h3-Open-UTIL/MP, such as ensemble coupling and Python application coupling, will be described in detail in the next section.

One of the features of h3-Open-UTIL/MP is that it uses the coupling library Jcup[2] as the basic software layer. Jcup is designed as a general-purpose library which provides the basic functions necessary for coupling, such as management of model components to be coupled and data exchange, and it is assumed that upper layers corresponding to individual environments will be implemented for actual use. For example, in an integrated land simulator (ILS), one of the application cases of Jcup, ILS coupling interface (ICI) is developed as an upper layer of Jcup, which provides necessary functions for ILS such as data monitoring and unit conversion[11]. As mentioned previously, h3-Open-UTIL/MP is intended to perform coupled computation for state-of-the-art computing environments as a part of h3-Open-BDEC. The h3-Open-BDEC is an interdisciplinary research project, and the project targets a wide range of applications, such as seismic models[4], [10] and weather/climate models. Therefore, the h3-Open-UTIL/MP must be able to flexibly support these wide range of models which have not only structured grid system but also unstructured grid system. For this purpose, it uses two input information: 1) the grid point index of each process of the model and 2) the table of the correspondence table between the grid point index of the sending and receiving models in the interpolation calculation and interpolation coefficients. The second information, i.e., the correspondence between the grid point index and the interpolation coefficients, is determined by the grid structure of the models to be coupled and the interpolation calculation algorithm. Therefore, the user of the coupler is required to calculate these values in advance depending on the individual environment. The concept about the input information and the interpolation calculation follows that of the base library Jcup, therefore, for details, readers are requested to refer to the Jcup reference[2]. The subroutines corresponding to 1) and 2) are shown in Tables 1 and 2. Subroutine hom_def_grid gives the grid point index for each model process. A single model component can have multiple grids, and each grid is identified by the argument grid_name. The subroutine hom_set_interpolation_table gives the corresponding table of send/receive grid point index and interpolation coefficients on interpolation calculation. This subroutine must be called by both sender and receiver model components. The arguments send_index, recv_index, and coef are optional arguments and are given by either the sender or the receiver. In addition to this information given as arguments to the subroutine, the rank number of each model determined at runtime is obtained from MPI. From this information, the grid point number to be sent by each process of the sending model and the rank number of the destination, as well as the grid point number to be received by the receiving model and the rank number of the source are calculated, and data exchange is performed appropriately. The interpolation calculation is also performed using the given grid point number and interpolation coefficient.

The information on the send/receive data is set in the configuration file. In the configuration file, four items are first set: the sending component name, sending grid name, receiving component name, and receiving grid name. Then, the send/receive data under this combination is set in items such as sender's data name, receiver's data name, and send/receive time interval.

There are only three types of subroutines to call for data exchange in the time integration loop, as shown in the Table 3. The subroutine hom_set_time provides the model name, current time, and $\Delta T$ for this time step. Inside this subroutine, data exchange and interpolation calculations are performed. The subroutine hom_put_data gives the data to the coupler. The given data is held in a buffer inside the coupler and sent to the other model at the timing when hom_set_time is called. The subroutine hom_get_data is a subroutine that retrieves data from the coupler. The argument is_recv_ok is a logical type variable that returns whether this time step is a data exchange step or not. The subroutines hom_put_data and hom_get_data may be called at any point in the time step loop after hom_set_time is called.

In the following sections, we present two examples of characteristic coupling programs implemented in the h3-Open-UTIL/MP: ensemble coupling and Python APP coupling.

\section{ENSEMBLE COUPLING}

Ensemble calculation performs many numerical simulations with slightly different initial and boundary conditions or model parameters. We can obtain more robust prediction results by using the statistical information presented by the ensemble calculation. This method is widely used in weather/climate simulations, for example, in the daily weather forecasts of the Japan Meteorological 


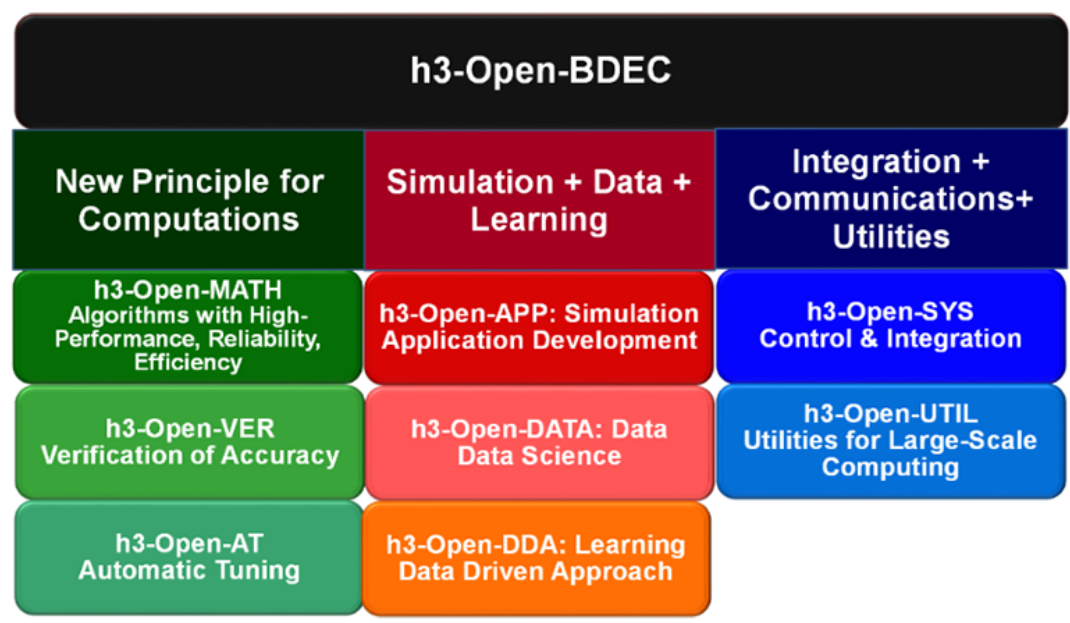

Figure 1: Structure of the h3-Open-BDEC project and software suite, via the h3-Open-BDEC webpage(http://h3-open-bdec.cc.utokyo.ac.jpjp/project.html).

Table 1: Grid index setting routines

\begin{tabular}{|c|c|c|c|}
\hline routine name & argument type & argument name & description \\
\hline \multicolumn{4}{|l|}{ hom_def_grid } \\
\hline & integer, intent(IN) & grid_index(:) & grid point index of earch process \\
\hline & character $\left(\right.$ len $\left.={ }^{*}\right)$, intent(IN) & comp_name & name of model component \\
\hline & character $\left(\right.$ len $\left.=^{*}\right)$, intent(IN) & grid_name & name of grid \\
\hline & integer, intent(IN) & $\mathrm{nz}$ & number of grid layers \\
\hline
\end{tabular}

Table 2: Routines of the interpolation index setting

\begin{tabular}{|c|c|c|c|}
\hline routine name & argument type & argument name & description \\
\hline \multicolumn{4}{|c|}{ hom_set_interpolation_table } \\
\hline & character $\left(\right.$ len $\left.=^{*}\right)$, intent(IN) & my_comp_name & name of my model \\
\hline & character $($ len $=*)$, intent(IN) & send_comp_name & name of send model \\
\hline & character $\left(\right.$ len $\left.=^{*}\right)$, intent(IN) & send_grid_name & name of send grid \\
\hline & character $\left(\right.$ len $\left.=^{*}\right)$, intent(IN) & recv_comp_name & name of receive model \\
\hline & character $($ len $=*)$, intent(IN) & recv_grid_name & name of receive grid \\
\hline & integer, intent(IN) & intpl_tag & identification tag of the table \\
\hline & integer, intent(IN) & send_index(:) & grid index of send model \\
\hline & integer, intent(IN) & recv_index(:) & grid index of receive model \\
\hline & $\operatorname{real}(\mathrm{kind}=8)$, intent(IN) & $\operatorname{coef}(:)$ & interpolation coefficient \\
\hline
\end{tabular}

Agency[1] and international future climate prediction experiments. Also, in ensemble-based data assimilation, we use the error covariance matrix obtained from the ensemble simulation. These ensemble calculations are multiple runs of a single model. On the other hand, there is also a desire to execute multiple simulations in a single run. In the high-resolution, large-ensemble data assimilation experiment conducted by Yashiro et al.[14], they designed a data assimilation system to execute a large number of ensemble members simultaneously to enable fast and scalable data assimilation calculation. There are also demands to get ensemble statistics while the simulation is running.

As mentioned previously, meteorological and climatic simulations often couple multiple models, so a coupled ensemble system is required to run multiple runs of the coupled model itself. Therefore, the h3-Open-UTIL/MP coupler has been designed and implemented to enable such coupled ensemble calculations. Fig. 2 shows the coupled ensemble pattern supported by h3-Open-UTIL/MP. The left side of the figure represents the case where multiple coupled models are executed simultaneously (many-to-many). In this case, the 
Table 3: Routines called in the time integration loop

\begin{tabular}{|c|c|c|c|}
\hline routine name & argument type & argument name & description \\
\hline \multicolumn{4}{|l|}{ hom_set_time } \\
\hline & character $\left(\right.$ len $\left.=^{*}\right)$, intent(IN) & comp_name & name of my model \\
\hline & integer, intent(IN) & time_array(6) & year,month,date,hour,minute,second \\
\hline & integer, intent(IN) & delta_t & $\Delta T$ \\
\hline \multicolumn{4}{|l|}{ hom_put_data } \\
\hline & character $\left(\right.$ len $\left.=^{*}\right)$, intent(IN) & data_name & name of data \\
\hline & $\operatorname{real}(\mathrm{kind}=8)$, intent$(\mathrm{IN})$ & data(:) or data(:,:) & data to send \\
\hline \multicolumn{4}{|l|}{ hom_get_data } \\
\hline & character(len=*), intent(IN) & data_name & name of data \\
\hline & real(kind=8), intent(INOUT) & data(:) or data(:,:) & data to receive \\
\hline & logical, intent(OUT) & is_recv_ok & flag whether receive is OK or NOT \\
\hline
\end{tabular}

statistics of each coupled simulation can be calculated at appropriate time intervals and output to a file or passed to an analysis program. The right side of the figure represents the case where one model B is coupled for multiple models A (many-to-one). In this coupling, at each data exchange between models $\mathrm{A}$ and $\mathrm{B}$, the average value of model $A$ is calculated and passed to model $B$, and the data of model B are broadcasted to each execution unit of model A. Although omitted in the figure, each model component itself is also parallelized by domain decomposition.

It is also assumed that the pattern and number of domain decompositions are the same for all ensembles. At runtime, the total number of processes in each model is given as an argument to mpiexec. For example, if the number of domain divisions for AppA is DA and the number of domain divisions for AppB is DB, the execution command is

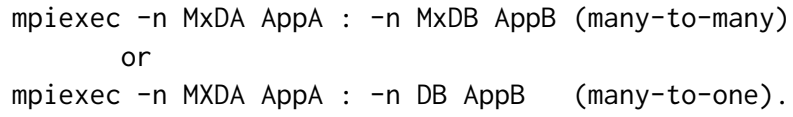

After the program is started, h3-Open-UTIL/MP allocates the MPI processes to each ensemble member according to the ensemble size given in the API of h3-Open-UTIL/MP. The types of MPI communicators generated and used for ensemble coupling by h3Open-UTIL/MP are shown in Fig. 3. In the figure, important for ensemble calculation are communicators consisting of all processes of the same model, represented by the blue line, and communicators consisting of the same region of the same model, represented by the red line. The former is used for global communication within the model, such as broadcasting various information in the initialization process of the coupling. The latter is used in the time integration process to calculate statistical values, such as the average, maximum, and minimum.

\section{PYTHON APPLICATION COUPLING}

\subsection{Overview of Python Coupling System}

Python is a programming language suitable for writing readable, clear, and logical code. It is used not only in HPC but also in many other fields. Furhter, there are many libraries available for various purposes, such as file I/O and data visualization. In particular, many ML frameworks, such as PyTorch and Keras, use Python. In particular, Python has become an indispensable language for ML implementation.

Typically, these Python applications are run standalone. However, even within these applications, running them coupled with other simulation models is often more convenient. For example, data visualization can be performed as a standalone application using the data output from the simulation model as input. However, when coupled with the simulation model, we can reduce the output file size and shorten the overall processing time. One of the most effective cases of coupled computation is the integration of simulation and ML. By substituting ML results for certain computational processes in the simulation, accuracy improvement and computation time reduction are expected. However, this method requires the interaction between simulation and ML, which cannot be achieved without coupled computation. We created a set of APIs for coupling Python applications as extensions to the h3-Open-UTIL/MP coupler based on this background.

Fig. 4 shows the program structure of the h3-Open-UTIL/MP coupler, including the Python API layer. The left block represents the program for the Fortran application. The API module, which the Fortran application uses to call the API subroutines, is at the top of the h3-Open-UTIL/MP coupler. The right block shows the program structure for Python applications. The Python APP imports the Python coupling API library h3opp and calls a set of procedures. Each procedure in h3opp calls the coupling API for Fortran. However, the API for Fortran is provided as a Fortran module file, which cannot be used by Python without modification. Therefore, a file h3open_py.f90 was created to define the subroutines without using the module, and the APIs of h3opp.py call the subroutines in h3open_py.f90.

To call Fortran routines from Python, we first need to convert the Fortran object files into library and import it into Python. To do this, we use the numpy function load_library. This code appears similar to the following.

h3oppf = np.ctypeslib.load_library("libh3opp.so", "./") 


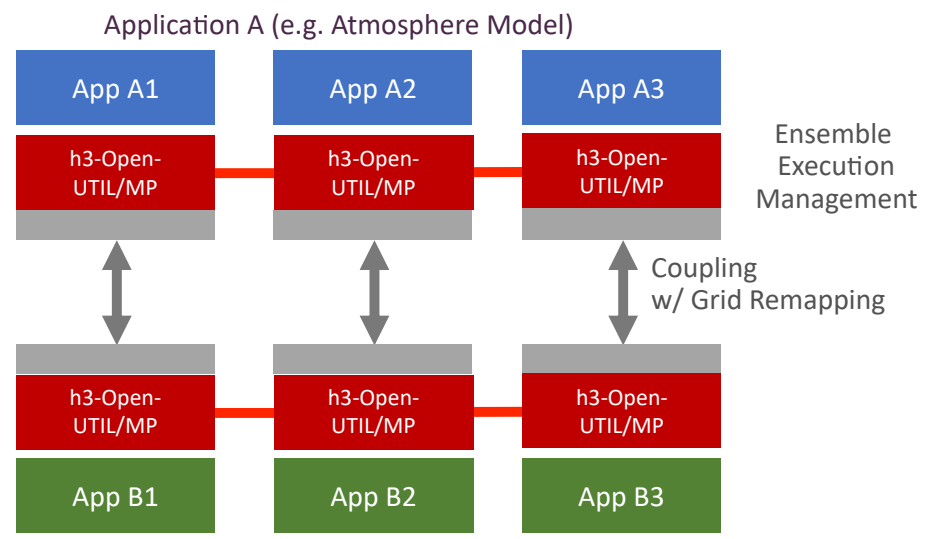

Application B (e.g. Ocean Model)

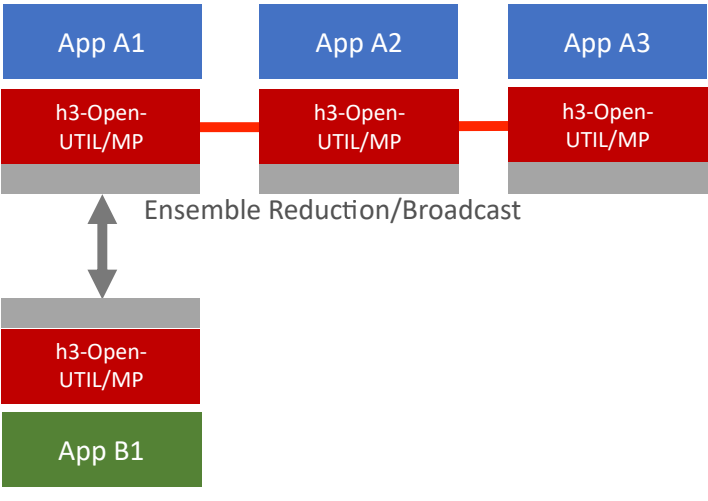

( $M \times A)+B$ execution

Figure 2: Ensemble coupling pattern of h3-Open-UTIL/MP. The left side of the figure represents a case where multiple coupled models are executed simultaneously, whereas the right side represents the case where one model B is coupled for multiple model A.

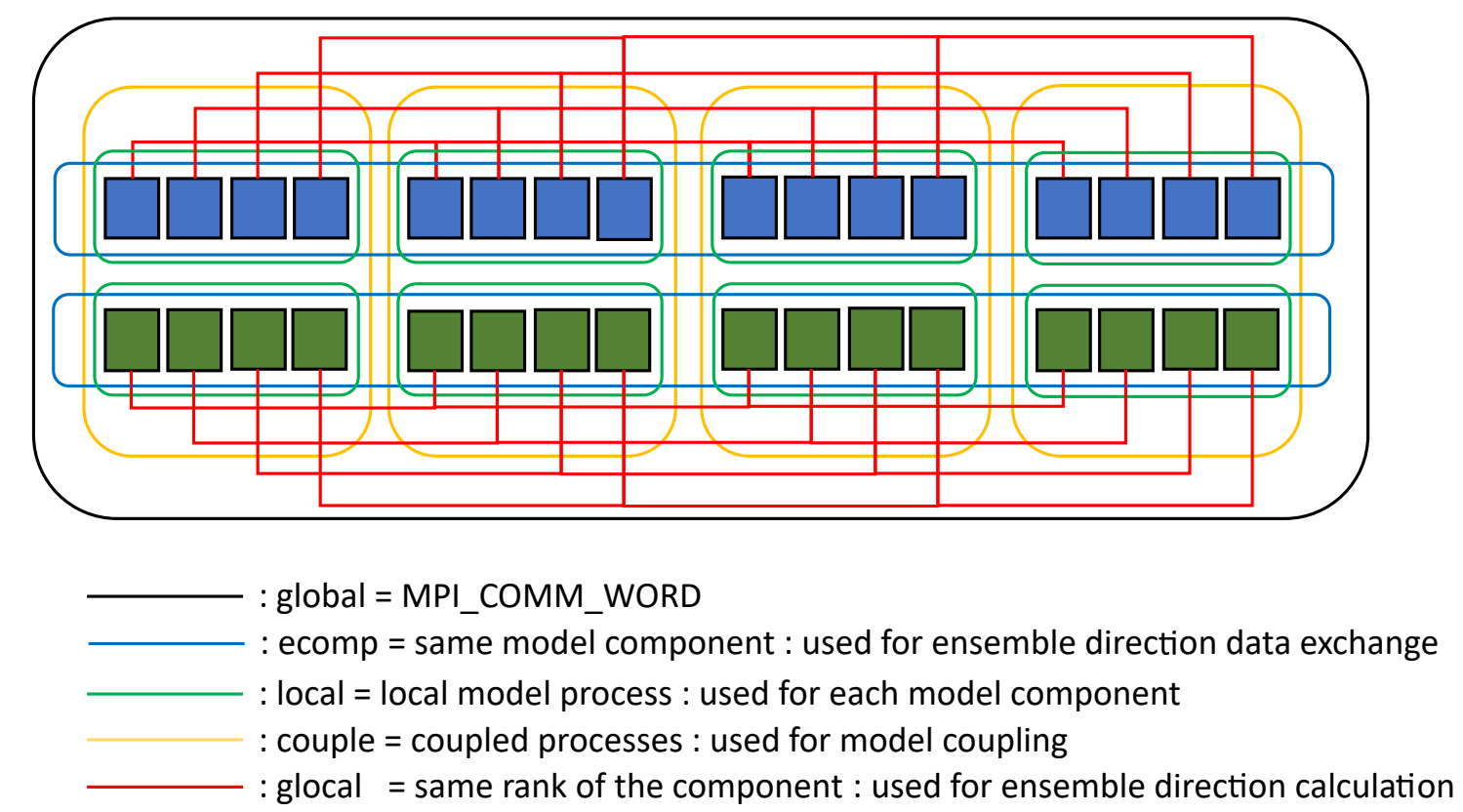

Figure 3: MPI communicators used for ensemble coupling.

The routines defined in the file h3open_py.f90 all have the bind(C) attribute and are called from Python as $\mathrm{C}$ functions. Fortran routines are call-by-reference, while $C$ functions are call-by-value. Therefore, when calling from Python, references must be explicitly used as an argument. For an example of a call from Python, the code for hop_def_grid, the Python version of the grid definition routine hom_def_grid described in Table 1, is shown in Fig. 5.
The left box contains the library loading and the functions used by hop_def_grid. Meanwhile, the right box show the code for the function hop_def_grid. This function first specifies the interface and return value of the Fortran routine. Then, the arguments of hop_def_grid are converted to pointers and passed to the Fortran routine. 


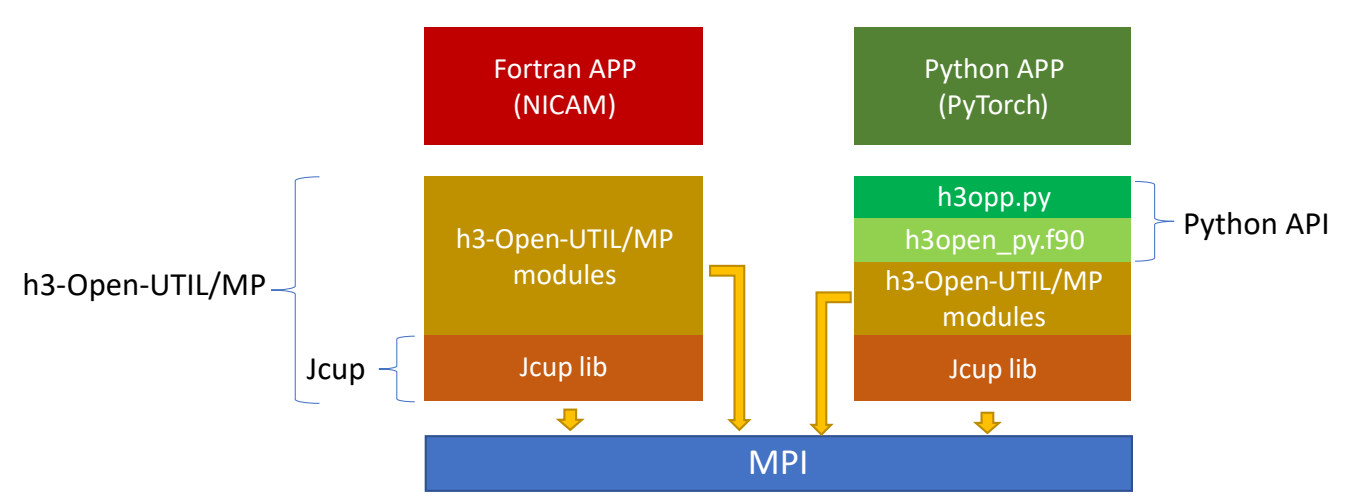

Figure 4: Program structure of the h3-Open-UTIL/MP coupler with the Python API layer.

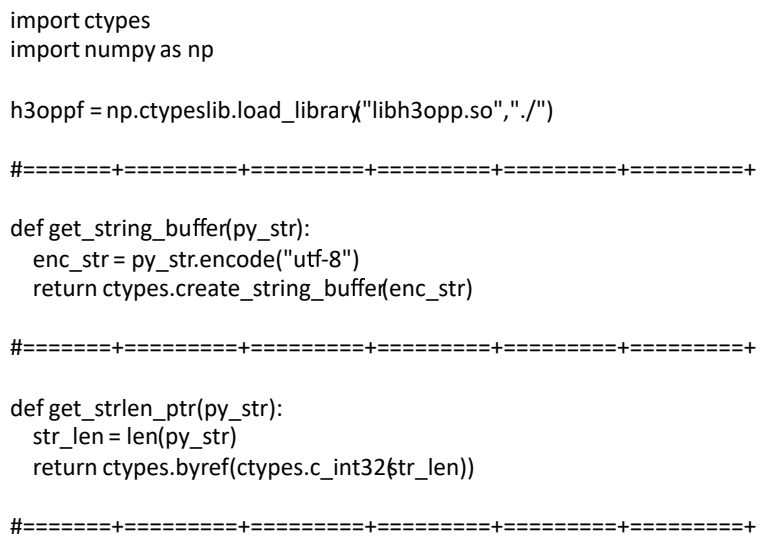

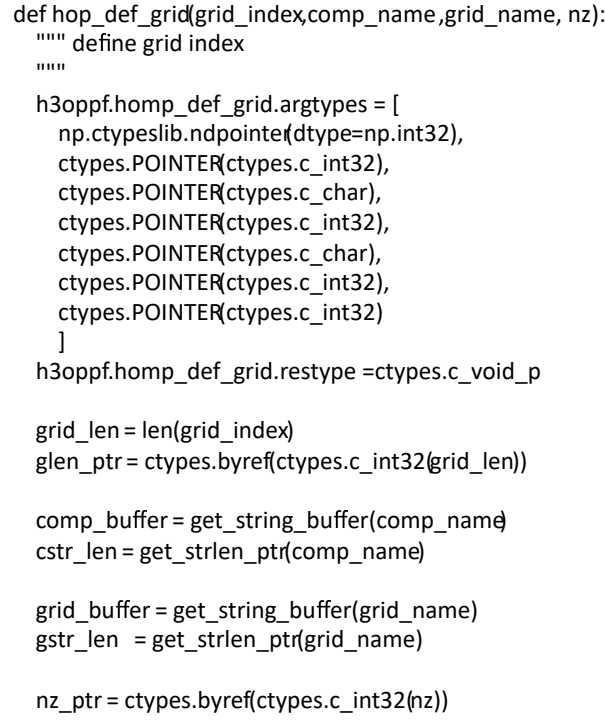

Figure 5: Sample code for calling a Fortran routine from Python.

\subsection{Implementation of Python Coupling Code}

This study selected ML as the Python application, as described in the next section. Typical ML libraries, such as PyTorch, do not support parallel computing with MPI. In h3-Open-UTIL/MP coupling, MPI is hidden within the coupling program, and parallelization is not necessarily required at the Python application level. However, processes related to coupled calculations, such as data exchange and interpolation calculations, can and should be performed in parallel. In addition, parallelization is expected to improve the performance for applications other than ML, such as IO and graphics. Therefore, Python applications in this study were also MPI parallelized. The library used for parallelization was mpi4py. All data exchange and other operations at the Python application level are performed using mpi4py functions. The problem here is the creation of a local communicator for the Python application and its introduction to the application. In h3-Open-UTIL/MP coupling, h3Open-UTIL/MP is responsible for the creation and management of communicators; the local communicator generated by h3-Open$\mathrm{UTLI} / \mathrm{MP}$ is given to the Fortran application. However, mpi4py uses its own communicator internally, and a local communicator cannot be provided from an external source. Therefore, we called a communicator splitting routine with MPI_COMM_WORLD as an argument in both the Fortran and Python applications, and then we used the split communicator as the local communicator used by mpi4py. In the environment where the authors actually ran the program, this method worked fine. However, note that splitting the communicator by calling MPI_comm_split and mpi4py split simultaneously is irregular and is not guaranteed to work.

To run PythonAPP and FortranAPP in parallel, simply give mpiexec multiple arguments as in the FortranAPP coupling case. An example of the execution is the following: 
mpiexec -np 16 FortranAPP : -np 4 Python3 PythonAPP

\subsection{NICAM and ML APP Coupling}

The example selected for the application of the Python coupling program is the NICAM and ML coupling. NICAM is a global atmospheric model employing an icosahedral grid system[12]. As NICAM can cover the entire globe with a nearly uniform grid, it is less constrained by CFL conditions than latitude-longitude grid models and can perform simulations over a wide range of grid spacing from several hundred kilometers to less than one kilometer. Therefore, multiple calculation schemes have been implemented to allow users to select the appropriate option depending on the resolution for the calculation of various meteorological phenomena, such as clouds and precipitation. For example, a cumulus parameterization scheme is used at a low resolution, where cumulus convection cannot be directly represented. Conversely, a scheme that directly represents cumulus clouds is used at a high resolution. The NICAM and ML coupling was implemented to alleviate the aforementioned limitations of the resolution-dependent computational scheme.

Fig. 6 shows a schematic of the NICAM-ML coupling. The sphere on the left side of the figure represents a high-resolution NICAM that directly represents cumulus convection. The blue bar represents the entire simulation sequence or the program code A certain physical process of a high-resolution NICAM (e.g., cloud physics) is represented by an orange square. The input and output (calculation results) of this calculation part (subroutine) are passed to the ML APP as training data. At this time, the coupler's remapping function converts the grid system of each training data from a highresolution NICAM to a low-resolution NICAM grid system. The results of ML will be output to a file. This process corresponds to Coupling Phase 1 in the figure. In Coupling Phase 2, the ML APP is coupled with the low-resolution NICAM represented by the sphere on the right side of the figure. In this coupling, the input data of the physical process of the low-resolution NICAM are passed to the ML APP. Then, the ML APP computes the output data from the training results of the high-resolution NICAM and the input data and returns it to the NICAM. Consequently, the results of cloud physics calculations that directly represent cumulus convection are learned for low-resolution grids that cannot directly represent cumulus convection. This enables the simulation that incorporates detailed physical representations of the high-resolution NICAM and is expected to accelerate the calculations.

\subsection{Case Study of the NICAM-ML Coupling}

The atmospheric model used in the case study (i.e., NICAM) is a global non-hydrostatic model with an icosahedral grid. The parameter Glevel expresses the number of horizontal grid points in NICAM, and the number of grid points quadruples when Glevel increases by one. The Glevel setting used in this experiment is Glevel07, indicating that the number of horizontal grid points is 163,840 and the grid spacing is about $50 \mathrm{~km}$. PyTorch, coupled to NICAM, has a Glevel05 grid that corresponds to a horizontal grid of 10,240 and a spacing of approximately $220 \mathrm{~km}$. The target of ML is the part that calculates the phase change of the water, the thermodynamical change, and precipitation, which is a subroutine called mp_driver in NICAM. In this subroutine, pressure, temperature, water contents, and other variables were used as input variables. We also calculated the tendency for each time step. We used the typical input variables and their tendencies as training data and trained the system to output the tendencies when the input variables were given. The six variables used in the study were total air density (rho), internal energy (ein), density of water vapor (rhoq), and their tendencies (d_rho, d_ein, d_rhoq). The variables for each region of the NICAM are sent to the Python APP via h3-Open-UTIL/MP. On the Python APP perspective, h3-Open-UTIL/MP converts each variable received from NICAM into a Glevel05 grid and passes it to Python APP. Then, the data passed to the Python APP are gathered to the root process. In the root process, training calculations are performed using PyTorch on the entire region data. In the following section, we discuss the results of ML. In this analysis, we used the Glevel05 data without spatial interpolation as a preliminary study to focus on the validity of the learning. We performed the analysis on the horizontal data of one vertical layer. The vertical layer used was the 20th layer. Figs. 7- 9 show the input and output variables (their tendencies). The input variables rho and ein show the influence of topography, and the spatial variation in values at low latitudes is small. Meanwhile, the input variable rhoq shows large variations, mainly at low latitudes. Tendencies presented on the right side of each figure show large fluctuations in all variables at the location corresponding to rhoq. This suggests that temporal changes in these physical quantities are strongly influenced by water vapor density.

ML was performed using the variables and tendencies in Figs. 7Fig. 9 as input and output variables, respectively. All variables were normalized with mean and standard deviation when they are input to ML. The ML method used here is a three-layer multi-layer perceptron (MLP). Fig. 10 shows the convergence of the learning process when the number of epochs per time step of the simulation is changed to 100,200 , and 300 . Note that the number of iterations is twice the number of epochs because we trained using two simulation time steps. Although there is a discontinuity in the center of each figure because the training data are replaced at each simulation step, the graphs converge uniformly, indicating that learning has no problem.

Figs. 11- 13 show the output variables (left panel) and the results of calculating the output variables from the learning results and input variables (right panel). The respective left figures are the same as the right figures in Figs. 7- 9. The rough distribution of all variables is well reproduced. However, compared to the left figures, the right figures show a smaller tone change, indicating that the reproduction of extreme values is not good. Fig. 14 shows a scatter plot of model calculation and ML for the three variables. The figure shows that most of the values are concentrated near zero, and only few are extreme. As shown in the previous figures, the extreme values are closer to 0 for the reproduced values by ML, and the slope of the distribution is smaller than 1 . The slope and intercept of the regression line and the correlation coefficient are summarized in Table 4 . The slopes of all variables are in the 0.5 range, which is smaller than the distribution of extreme values. This is thought to be because there is a problem with the reproducibility of values near 0 , which are relatively numerous. As shown in Fig. 14, the distribution 


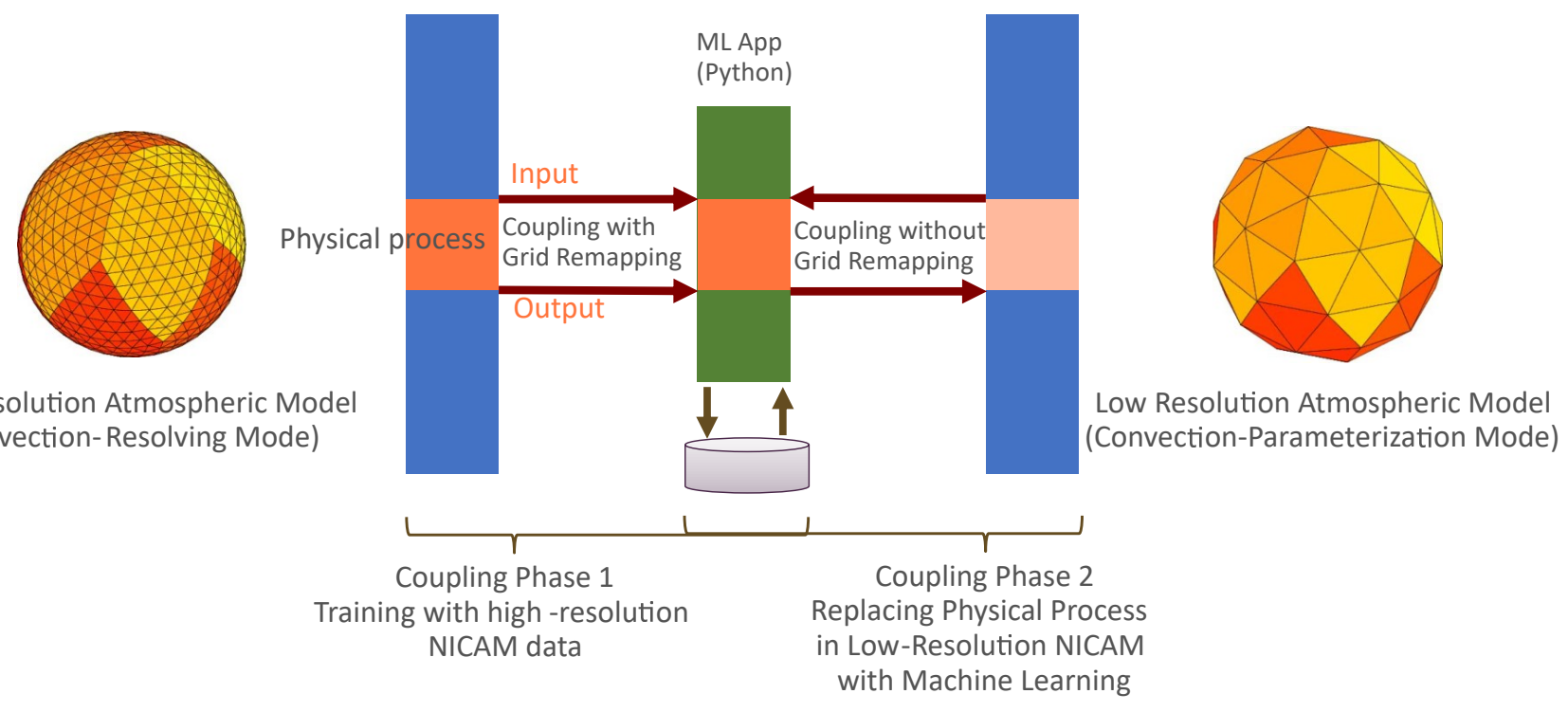

Figure 6: Schematics of the NICAM and ML framework coupling. Coupling Phase 1 is the training phase wherein training the ML is performed using the NICAM data. Coupling Phase 2 is the computation phase wherein the training results are used to compute the physical processes in NICAM.
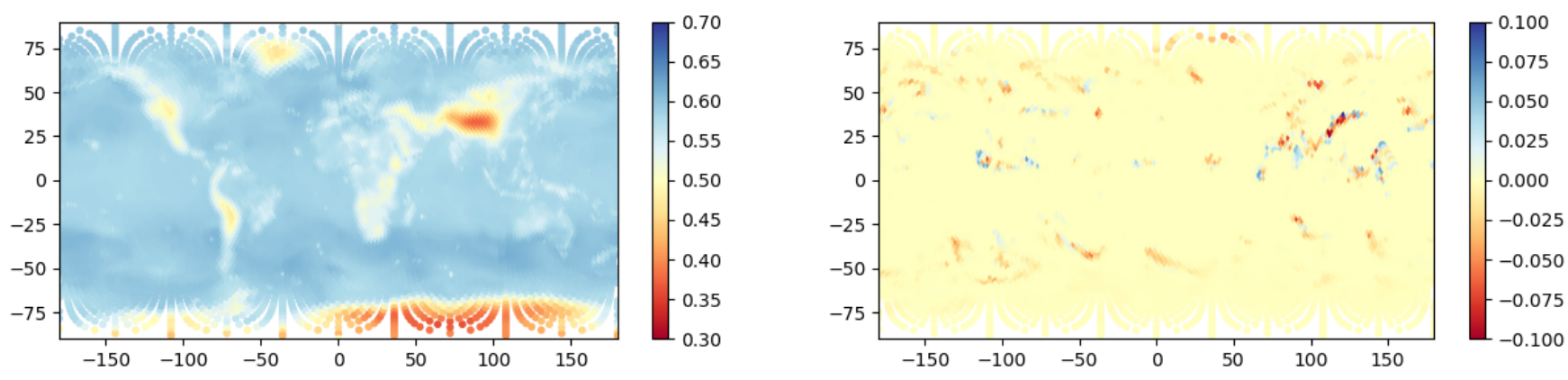

Figure 7: Horizontal distribution of the total air density $\left[\mathrm{kg} / \mathrm{m}^{3}\right]$ (left panel, referred in the text as rho) and its tendency $\left[\mathrm{kg} / \mathrm{m}^{3} / \mathbf{t} * 10^{6}\right]$ (right panel, referred in the text as d_rho).

appers different after the zero lines. This is probably because ML failed to learn the precise saturation water vapor pressure from the temperature and density conditions or the difference between liquid water and ice.

Table 4: Correlation

\begin{tabular}{cccc}
\hline variable & slope & intercept & correlation coefficient \\
\hline d_rho & 0.53847887 & -0.00017136 & 0.6194595 \\
d_ein & 0.58866578 & -0.00019053 & 0.6769882 \\
d_rhoq & 0.56042855 & 0.000008773 & 0.6494912 \\
\hline
\end{tabular}

\section{CONCLUSION}

In this study, the h3-Open-UTIL/MP coupler was developed as part of the h3-Open-BDEC project. This coupler has features adapted to the h3-Open-BDEC project's goals of large-scale data handling and ML, such as ensemble coupling and Python application coupling. However, there is still room for significant improvement in terms of ML reproducibility and computational performance. As the reproducibility is not directly related to the coupler, we only discuss this briefly. As mentioned previously, there are several factors that reduce the reproducibility. The problem of the slope of the regression line caused by data near the zero point might be solved by weighting the values near the zero point in the preprocessing stage. For the difference in distribution around the zero point, it may be effective to incorporate appropriate variables into the training. Furthermore, note that these data have a spatial (temporal) structure. The MLP used in this study is an algorithm that learns using only point-by-point relationships and does not reflect spatial (or temporal) structure. Therefore, we can expect better reproducibility by 

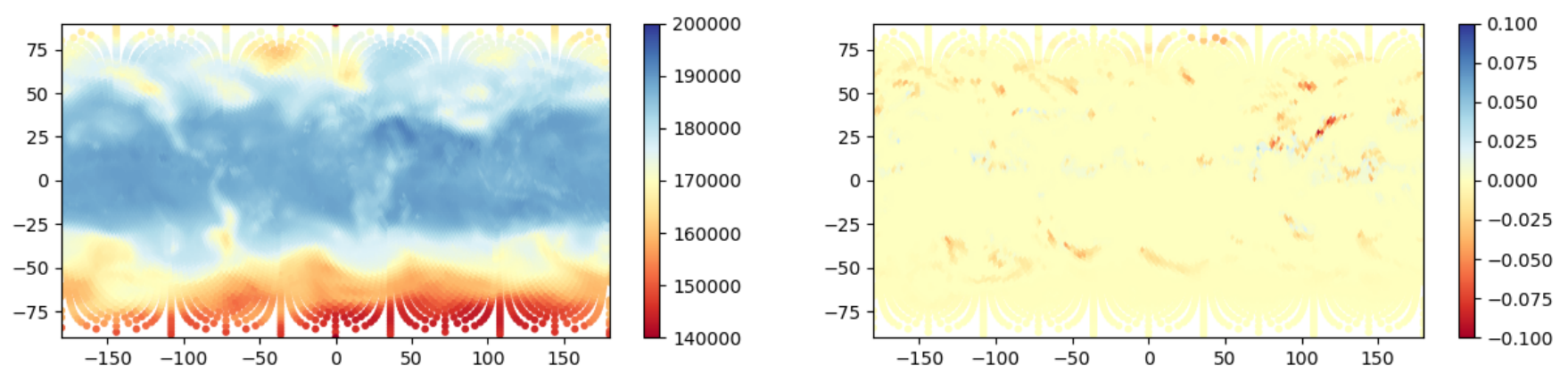

Figure 8: Horizontal distribution of the internal energy $[\mathrm{J} / \mathrm{kg}]$ (left panel, referred in the text as ein) and its tendency[J/kg/t $]$ (right panel, referred in the text as d_ein).
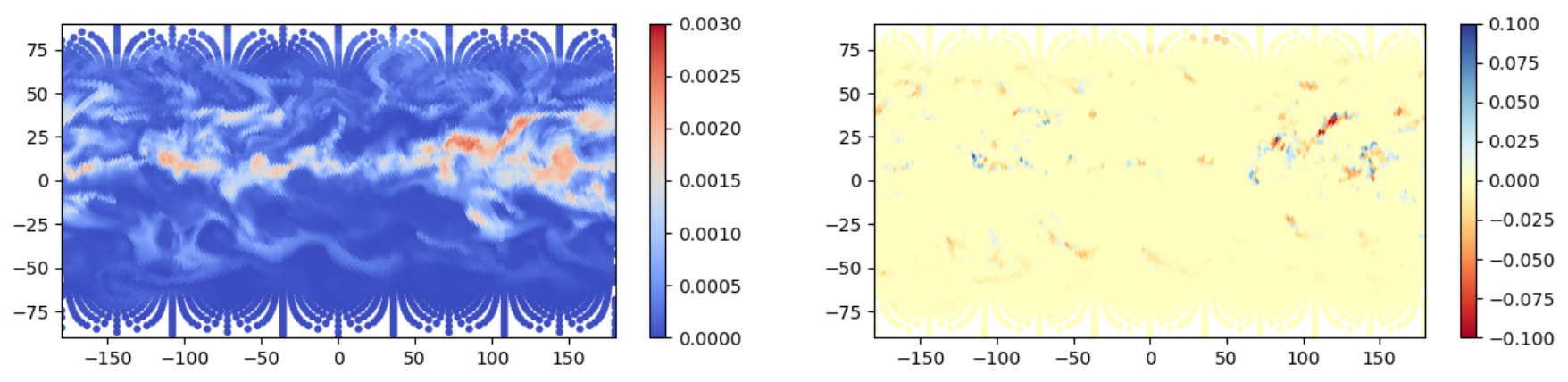

Figure 9: Horizontal distribution of the density of water vapor[ $\left.\mathrm{kg} / \mathrm{m}^{3}\right]$ (left panel, referred in the text as rhoq) and its tencench $\left[\mathrm{km} / \mathrm{m}^{3} / \mathbf{t} * 10^{6}\right]$ (right panel, referred in the text as d_rhoq)
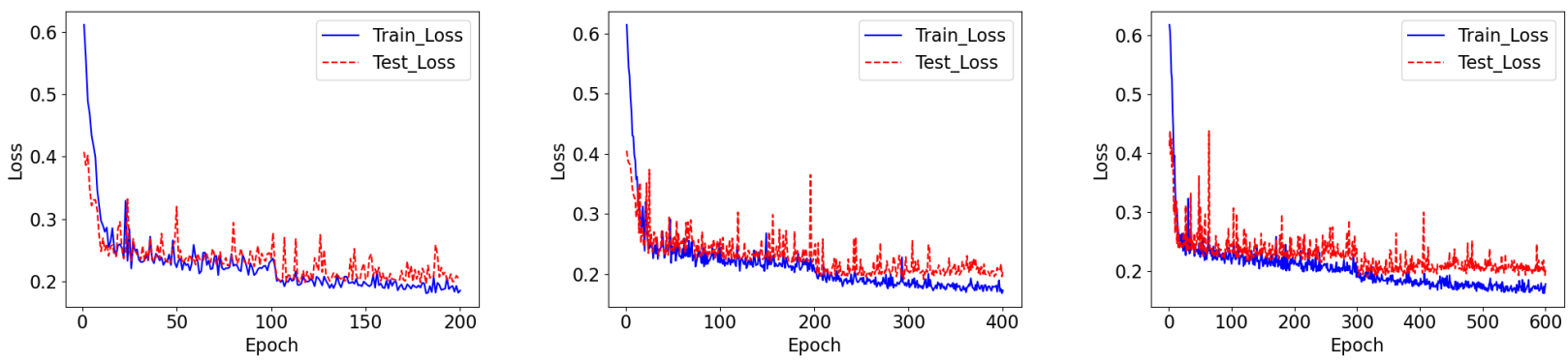

Figure 10: The convergence of the learning process when the number of epochs per time step is changed to 100 (left), 200 (middle), and 300 (right). Although there is a discontinuity in the center of each figure because the training data are replaced at each simulation step, the graphs converge uniformly, indicating that learning has no problem.

using an algorithm that can reflect the spatio-temporal structure, such as CNN, instead of MLP.

In addition to the reproduction accuracy mentioned, there is also the issue of computational performance. We performed the aforementioned coupled calculations on Oakforest-PACS at the Information Technology Center, the university of Tokyo. The performance bottleneck was the learning calculation of the ML framework. One method to solve this bottleneck is to perform the computation in an environment suitable for ML. Many ML frameworks, such as PyTorch, support GPUs; hence, we can expect significant performance improvements by using GPUs for computation. Meanwhile, most of the simulation programs coupled with ML frameworks are not GPU-compatible and are tuned to achieve high performance on conventional high-performance computers. Therefore, for the coupled computation of the simulation program and the ML framework, the most suitable environment is where we can run the simulation 

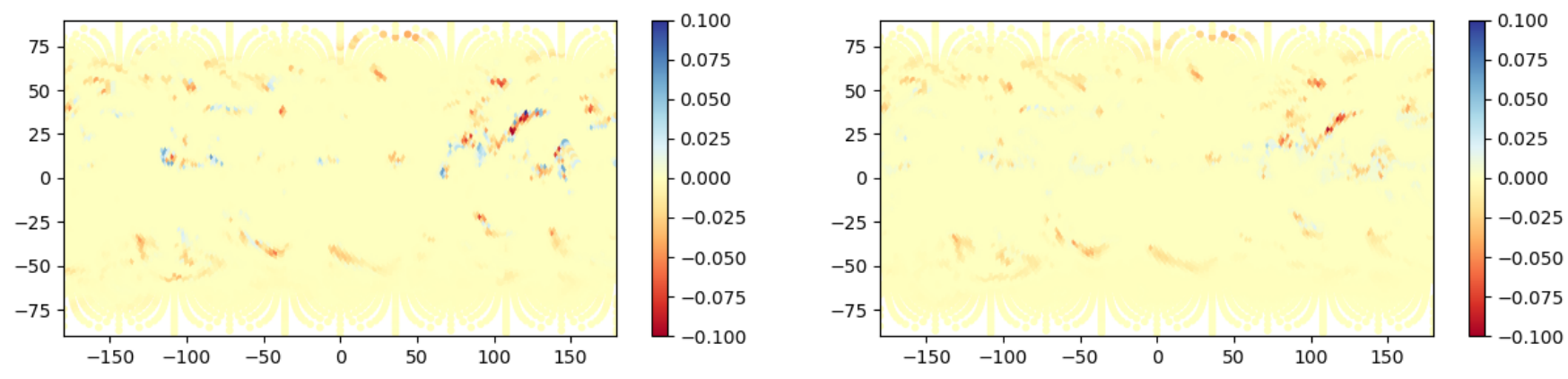

Figure 11: Horizontal distribution of the tendency of the total air density[ $\left.\mathrm{kg} / \mathrm{m}^{3} / \mathrm{t} * 10^{6}\right](=\mathbf{d}$-rho), left panel:model-calculated value, and right panel:reproduced value by $M L$.
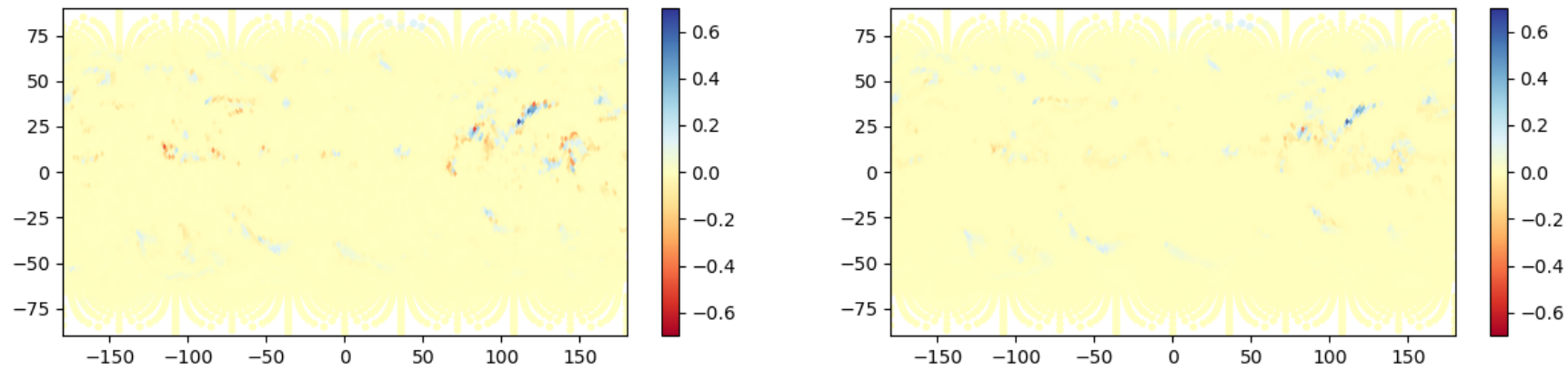

Figure 12: Horizontal distribution of the tendency of the internal energy $[\mathrm{J} / \mathrm{kg} / \mathrm{t}]\left(=\mathrm{e} \_\right.$ein), left panel:model-calculated value, and right panel:reproduced value by $M L$.
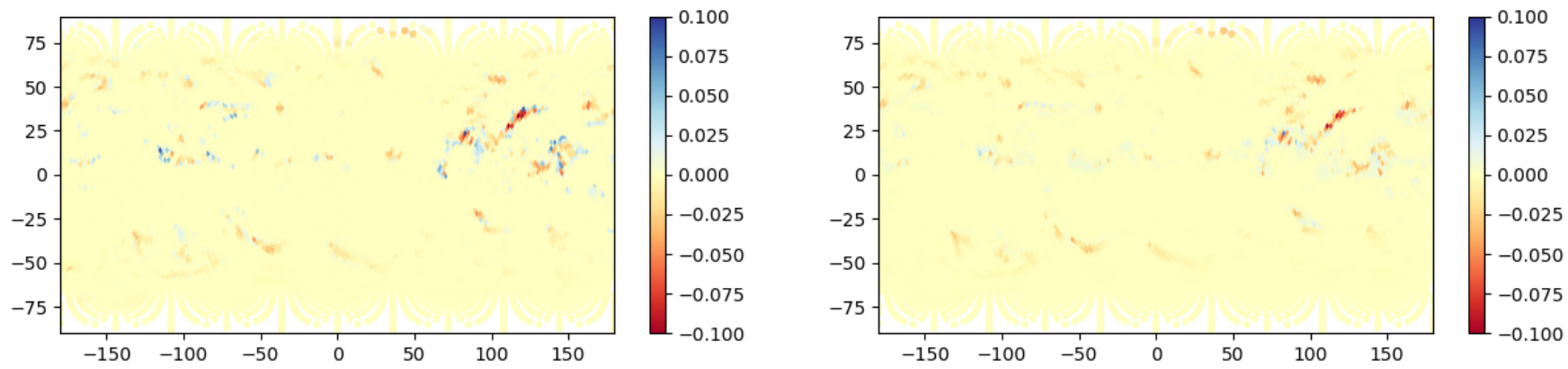

Figure 13: Horizontal distribution of the tendency of the density of water vapor $\left[\mathrm{km} / \mathrm{m}^{3} / \mathbf{t} * 10^{6}\right](=\mathbf{d}$ _rhoq), left panel:modelcalculated value, and right panel:reproduced value by ML.

program on a conventional HPC and the ML framework on a GPU machine in parallel.

Information Technology Center, The university of Tokyo began using the Wisteria/BDEC-01 supercomputer in July 2021. According to the Center's documentation[7], the system configuration is introduced as follows.

Wisteria/BDEC-01 is a hybrid system, including two groups of compute nodes: Simulation Nodes (Odyssey) and Data/Learning Nodes (Aquarius). Simulation nodes group (Odyssey) consists of 7,680 compute nodes equipped with Fujitsu Limited's FUJITSU Processor A64FX. Data/Learning nodes group (Aquarius) consists of 45 compute nodes with Intel 3rd Generation Xeon Scalable Processors (code name: Ice Lake) and NVIDIA A100 Tensor Core GPUs. 

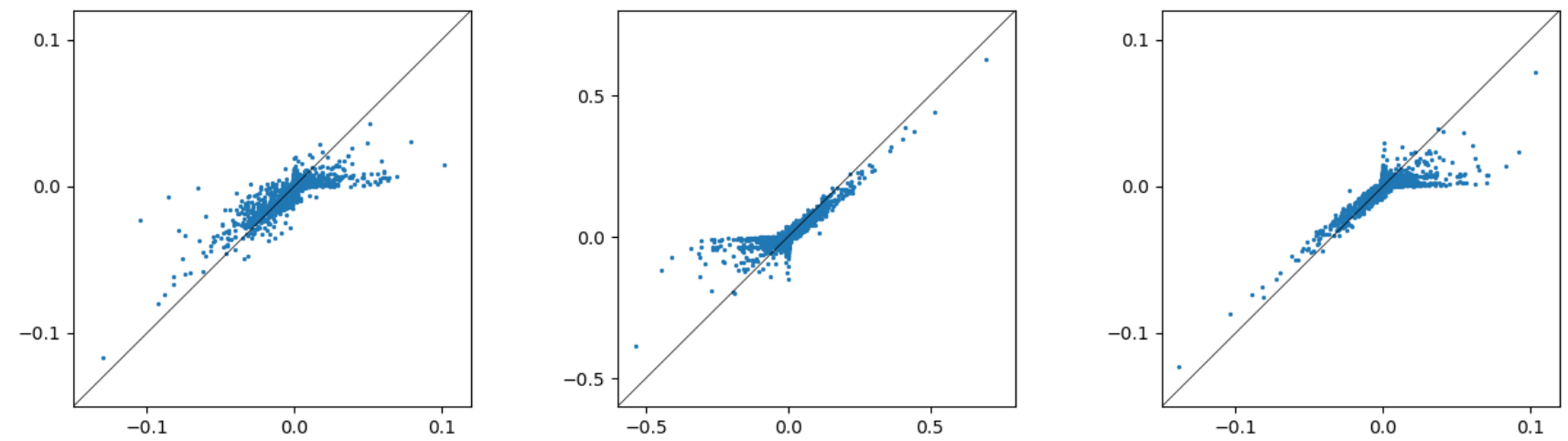

Figure 14: Scatter plots of the model values and calculated values(left:d_rho, middle:d_ein, right:d_rhoq)

Therefore, if coupled computation is possible between Odyssey and Aquarius, it is an ideal environment for coupling a simulation model and a ML framework. However, the MPI environment for coupling between Odyssey and Aquarius is not, at least for now, provided. Therefore, as part of the h3-Open-BDEC project, another team (h3-Open-SYS team) is developing WaitIO-Socket, a library for communication between heterogeneous machines. WaitIO-Socket implements communicator generation and data exchange procedures etc. for heterogeneous communication. The three main data sending/receiving procedures provided by WaitIOSocket are waitio_isend, waitio_irecv, and watio_wait, which can be used in the same way as the similar MPI procedures MP_isend, MPI_irecv, and MPI|_wait. On the other hand, the group of MPI routines that h3-Open-UTIL/MP uses between models(= between heterogeneous machines), consists of seven types: MPI_Bcast, MPI_gather, MPI_Allreduce, MPI_Reduce, MPI_isend, MPI_irecv, and MPI_waitall. Therefore, by substituting these groups of routines with the WaitIOSocket group of routines, coupling between heterogeneous machines come to be possible.

The concept of the entire program structure is as shown in Fig.15. MPI is used for intra-machine communication, WaitIO-Socket for inter-machine communication, and both are used together for global communication. On top of these programs, a wrapper layer that emulates MPI routines is created, so that the coupler can use the existing calls of MPI routines with almost no modification. The implementation of WaitIO-Socket and its application to h3-OpenUTIL/MP is in progress, and we plan to report on these details in our presentation at the conference.

\section{ACKNOWLEDGMENTS}

This work was supported by JSPS KAKENHI Grant Number JP19H05662.

\section{REFERENCES}

[1] Japan Meteorological Agency. 2021. 7MA Numerical Weather Prediction. Retrieved Jul 25, 2021 from https://www.jma.go.jp/jma/jma-eng/jma-center/nwp/nwp-top. htm

[2] Takashi Arakawa, Takahiro Inoue, Hisashi Yashiro, and Masaki Satoh. 2020 Coupling library Jcup3: its philosophy and application. Progress in Earth and

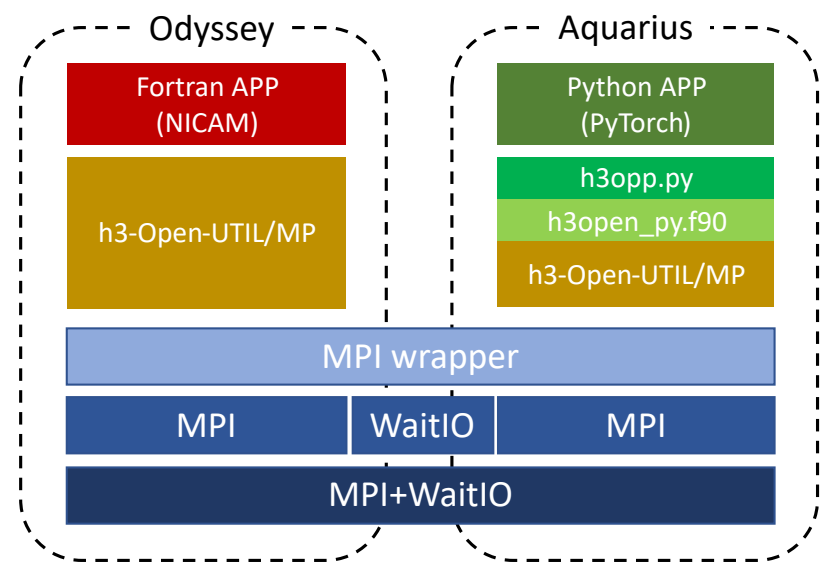

Figure 15: Program structure for inter-machine (Odyssey+Aquarius) coupling.

Planetary Science 7, 6 (2020). https://doi.org/10.1186/s40645-019-0320-z

[3] Anthony Craig, Sophie Valcke, and Laure Coquart. 2017. Development and performance of a new version of the OASIS coupler, OASIS3-MCT_3.0. Geoscientific Model Development 10, 9 (2017), 3297-3308. https://doi.org/10.5194/gmd-103297-2017

[4] Takashi Furumura, Takuto Maeda, and Atsuki Oba. 2019. Early Forecast of Long-Period Ground Motions via Data Assimilation of Observed Ground Motions and Wave Propagation Simulations. Geophysical Research Letters 46, 1 (2019), 138-147. https://doi.org/10.1029/2018GL081163 arXiv:https://agupubs.onlinelibrary.wiley.com/doi/pdf/10.1029/2018GL081163

[5] Moritz Hanke, Rene Redler, Teresa Holfeld, and Maxim Yastremsky. 2016. YAC 1.2.0: new aspects for coupling software in Earth system modelling. Geoscientific Model Development 9, 8 (2016), 2755-2769. https://doi.org/10.5194/gmd-9-27552016

[6] C. Hill, C. DeLuca, V. Balaji, M. Suarez, and A. DaSilva. 2004. The architecture of the earth system modeling framework. Computing in Science and Engineering 6, 1 (2004), 12-28. https://doi.org/10.1109/MCISE.2004.1255817

[7] The University of Tokyo Information Technology Center. [n.d.]. Introduction to the Wisteria/BDEC-01. Retrieved Jul 31, 2021 from https://www.cc.u-tokyo.ac.jp/ en/supercomputer/wisteria/system.php

[8] Michio Kawamiya, Tomohiro Hajima, Kaoru Tachiiri, Shingo Watanabe, and Tokuta Yokohata. 2020. Two decades of Earth system modeling with an emphasis on Model for Interdisciplinary Research on Climate (MIROC). Progress in Earth and Planetary Science 7, 64 (Aug. 2020). https://doi.org/10.1186/s40645-02000369-5 
[9] Kengo Nakajima. 2019. h3-Open-BDEC presentations. Retrieved Jul 25, 2021 from http://nkl.cc.u-tokyo.ac.jp/h3-Open-BDEC

[10] Kengo Nakajima, Hiroya Matsuba, Toshihiro Hanawa, Takashi Furumura, Hiroshi Tsuruoka, and Hiromichi Nagao. 2021. Integration of 3D Earthquake Simulation \& Real-Time Data Assimilation on h3-Open-BDEC. SIAM Conference on Computational Science \& Engineering (CSE21).

[11] Tomoko Nitta, Takashi Arakawa, Misako Hatono, Akira Takeshima, and Kei Yoshimura. 2020. Development of Integrated Land Simulator. Progress in Earth and Planetary Science 7, 68 (2020). https://doi.org/10.1186/s40645-020-00383-7

[12] Masaki Satoh, Hirofumi Tomita, Hisashi Yashiro, Hiroaki Miura, Chihiro Kodama, Tatsuya Seiki, Akira Noda, Yohei Yamada, Daisuke Goto, Masahiro Sawada, Takemasa Miyoshi, Yosuke Niwa, Masayuki Hara, Tomoki Ohno, Shin-ichi Iga, Takashi Arakawa, Takahiro Inoue, and Hiroyasu Kubokawa. 2014. The Non-hydrostatic Icosahedral Atmospheric Model: description and development. Progress in Earth and Planetary Science 1 (10 2014), 18. https://doi.org/10.1186/s40645-014-0018-1

[13] G. Theurich, C. DeLuca, T. Campbell, F. Liu, K. Saint, M. Vertenstein, J. Chen R. Oehmke, J. Doyle, T. Whitcomb, A. Wallcraft, M. Iredell, T. Black, A. M. da Silva, T. Clune, R. Ferraro, P. Li, M. Kelley, I. Aleinov, V. Balaji, N. Zadeh, R.
Jacob, B. Kirtman, F. Giraldo, D. McCarren, S. Sandgathe, S. Peckham, and R. Dunlap, IV. 2016. The Earth System Prediction Suite: Toward a coordinated U.S. modeling capability. Bull. Amer. Meteorol. Soc. 97, 7 (2016), 1229-1247. https: //doi.org/10.1175/BAMS-D-14-00164.1

[14] Hisashi Yashiro, Koji Terasaki, Yuta Kawai, Shuhei Kudo, Takemasa Miyoshi, Toshiyuki Imamura, Kazuo Minami, Hikaru Inoue, Tatsuo Nishiki, Takayuki Saji, Masaki Satoh, and Hirofumi Tomita. 2020. A 1024-Member Ensemble Data Assimilation with 3.5-Km Mesh Global Weather Simulations. In SC20: International Conference for High Performance Computing, Networking, Storage and Analysis. 1-10. https://doi.org/10.1109/SC41405.2020.00005

[15] Hiromasa Yoshimura and Seiji Yukimoto. 2008. Development of a Simple Couple (Scup) for Earth System Modeling. Papers in Meteorology and Geophysics 59 (2008), 19-29. https://doi.org/10.2467/mripapers.59.19

[16] Seiji Yukimoto, Akira Noda, Akio Kitoh, Masato Sugi, Yoshiteru Kitamura, Masahiro Hosaka, Kiyotaka Shibata, Shuhei Maeda, and Takao Uchiyama. 2001. The New Meteorological Research Institute Coupled GCM (MRI-CGCM2). Papers in Meteorology and Geophysics 51, 2 (Feb. 2001), 47-88. https://doi.org/10.2467/ mripapers.51.47 